\title{
Experimental Design for Single Point Diamond Turning of Silicon Optics
}

\author{
Debra A. Krulewich
}

June 16, 1996

This is an informal report intended primarily for internal or limited external distribution. The opinions and conclusions stated are those of the author and may or may not be those of the Laboratory.

Work performed under the auspices of the U.S. Department of Energy by the Lawrence Livermore National Laboratory under Contract W-7405-Eng-48. 


\section{DISCLAIMER}

This document was prepared as an account of work sponsored by an agency of the United States Government. Neither the United States Government nor the University of California nor any of their employees, makes any warranty, express or implied, or assumes any legal liability or responsibility for the accuracy, completeness, or usefulness of any information, apparatus, product, or process disclosed, or represents that its use would not infringe privately owned rights. Reference herein to any specific commercial product, process, or service by trade name, trademark, manufacturer, or otherwise, does not necessarily constitute or imply its endorsement, recommendation, or favoring by the United States Government or the University of California. The views and opinions of authors expressed herein do not necessarily state or reflect those of the United States Government or the University of California, and shall not be used for advertising or product endorsement purposes.

This report has been reproduced directly from the best available copy.

Available to DOE and DOE contractors from the Office of Scientific and Technical Information P.O. Box 62, Oak Ridge, TN 37831

Prices available from (615) 576-8401, FTS 626-8401

Available to the public from the National Technical Information Service

U.S. Department of Commerce 5285 Port Royal Rd., Springfield, VA 22161 


\title{
EXPERIMENTAL DESIGN FOR
}

\section{SINGLE POINT DIAMOND TURNING OF SILICON OPTICS}

\author{
Debra A. Krulewich \\ Engineer \\ Mechanical Engineering Department \\ Materials and Manufacturing Engineering Division \\ Precision Systems and Manufacturing Group \\ Lawrence Livermore National Laboratory \\ University of California \\ Livermore, CA 94551
}

October 15, 1996 


\section{Overview of Experimental Design}

Experimental design is a systematic method of selecting optimal settings for a limited set of experiments. Using experimental design, we can extract more information from fewer runs. This information is easier to analyze in comparison to the traditional experimental approaches. Intelligently designed experiments have a great advantage when analyzing the effects of many factors on a response. In this case, it is often not economical to run the full matrix of experiments. Experimental design procedures provide an efficient methodology for the selection of the optimum subset of experiments.

Traditional methods usually involve varying a single factor until the optimum results are attained. This factor then remains fixed for the remainder of the experiment while the next factor is varied. A problem arises in that the effect of a single factor can be influenced by the settings of other factors, so the settings interact. By varying a single factor at a time, we can only be sure that we have located a local optimum location. Furthermore, it is difficult to assess the statistical significance of each factor. For example, we may think that we have obtained an optimum setting by observing the response, while the majority of the response was actually caused by noise rather than varying the single factor.

During the experimental design process, we make informed assumptions at the onset. On the surface, this may appear to be a disadvantage. However, unavoidable assumptions are inadvertently made during the traditional approach. During experimental design the assumptions are intentional, and the purpose of the experiment is to support or disprove these assumptions.

Experimental design consists of the following stages:

1. Define the problem.

2. Determine the objective of the experiment.

3. Generate an a priori model.

4. Create the experimental design.

5. Analyze the data.

\section{Problem Definition}

To define the problem, we must first identify all the inputs and outputs of the system. We also select the range, or settings of the input factors. The inputs can be either qualitative or quantitative. A qualitative variable has discrete settings, such as the type of cutting tool. A quantitative variable has a numerical value and is usually continuous, such as the surface velocity. 
We must also take a first guess at the factors that we expect to interact. Interactions occur when the response caused by one factor is affected by the settings of another factor. Intelligent assumptions should be made based on understanding of the system. These assumptions will effect the experimental design. However, during the data analysis, we will test these assumptions and possibly add additional experiments based on our results. If we choose to include the effects of many interactions, this will greatly increase the number of required experiments. Also, the number of interactions we choose to study will also be influenced by the objective of the experiment.

\section{Experimental Objectives}

The experimental objective can range from simply identifying which factors affect a process to optimizing performance or formulating a predictive model. In order to develop a sound predictive model, often many experiments are required. Usually we begin the experimental design process by selecting the significant factors during a screening experiment. Then more in depth experiments are performed with only the significant factors to develop a predictive model or optimize the performance.

\section{1 Screening}

The objective of screening experiments is to reduce a large number of potentially important factors to those that are most significant. Often it is not economically practical to perform every possible combination of factor settings. In these situations screening is a very useful tool.

In order to reduce the number of experiments, usually we study only two states (a high and a low) for each factor. With only two states, it is only possible to observe linear effects of the factor on the response. Since our objective during screening is to determine significant factors rather than to develop predictive capabilities, this is usually sufficient. Later, during response surface methodology, additional settings for each factor are added.

To further reduce the number of experiments, often only first order effects are studied. However, it is not possible to assess the effects of the interactions. Furthermore, the interaction effects will be coupled to other first order effects and may cause erroneous results.

As an alternative, we can consider all or some of the second order interactions. It is usually safe to assume that third and higher order interactions are insignificant during a screening experiment. At this stage it is useful to have an understanding of the physical system being studied so an intelligent decision can be made. 


\subsection{Response Surface Methodology}

The objective of response surface methodology (RSM) is to empirically determine a predictive model for the process. Usually RSM follows a screening experiment where the significant factors were identified and the a priori model has been refined. With the additional knowledge and confidence in the model provided by the screening experiment, it is now possible to increase the number of settings on each factor to study non-linear effects of the factors while still keeping the total number of experiments under a practical limit. Usually RSM designs are associated with quadratic models. In the limited space of the experiment, usually quadratic models can adequately fit the region.

It is still not necessary to run the full matrix of experiments to determine the predictive model. The refined model will aid in the selection of the experimental design. Also we can perform predictive models with both qualitative and quantitative factors, although the type of experimental design we choose will be different.

\subsection{Taguchi Method}

The Taguchi method is mentioned here for completeness, although it does not apply to the particular case under study of single point diamond turning of silicon optics. The Taguchi method was developed by Dr. Genichi Taguchi, a Japanese engineer interested in quality control of manufacturing processes. The goal of the Taguchi method is to increase productivity while decreasing cost. Traditional manufacturing methods identify a process and the factors that control the process. To produce desired response, all the process factors are tightly controlled. In contrast, the Taguchi method identifies factors that are difficult or expensive to control as noise factors and the remaining factors to be control factors. A planned set of experiments is performed that aids in the selection of the control factor settings that is least sensitive to the varying uncontrolled factors. The Taguchi method also takes into account the cost of the process.

\section{Types of Experimental Designs}

There are many methods of selecting the optimum set of experiments. The selection of the method is based on the assumptions about the process and the objective of the experimentation.

\section{1 Full Factorial Designs}

A full factorial design contains the complete matrix of every possible experiment. The full factorial design requires many experimental runs and is often not economically practical. Although the results from a full factorial design are more intuitive, the full set of experiments is rarely necessary, especially for a screening experiment. For example, for an 
experiment with five factors with two settings each, a full factorial design dictates that $2^{5}$, or 32 runs must be performed. Even when it is feasible to perform the full factorial set of experiments, literature still recommends first running a half fractional factorial design first. The results of the reduced set may alter the settings or reduce the number of required remaining experiments.

If the goal of the experiment is to study the effects and all interactions, full factorial design provides exactly the number of degrees of freedom required to fit the full model, with no remaining degrees of freedom to assess the fit. This is equivalent to fitting a third order polynomial exactly through four points. The fitted polynomial will exactly fit the four points. There are two approaches to solve this problem. The first approach is to perform replicated runs, although this requires additional experiments. The second solution is to collapse the analysis on factors that seem insignificant after the fit.
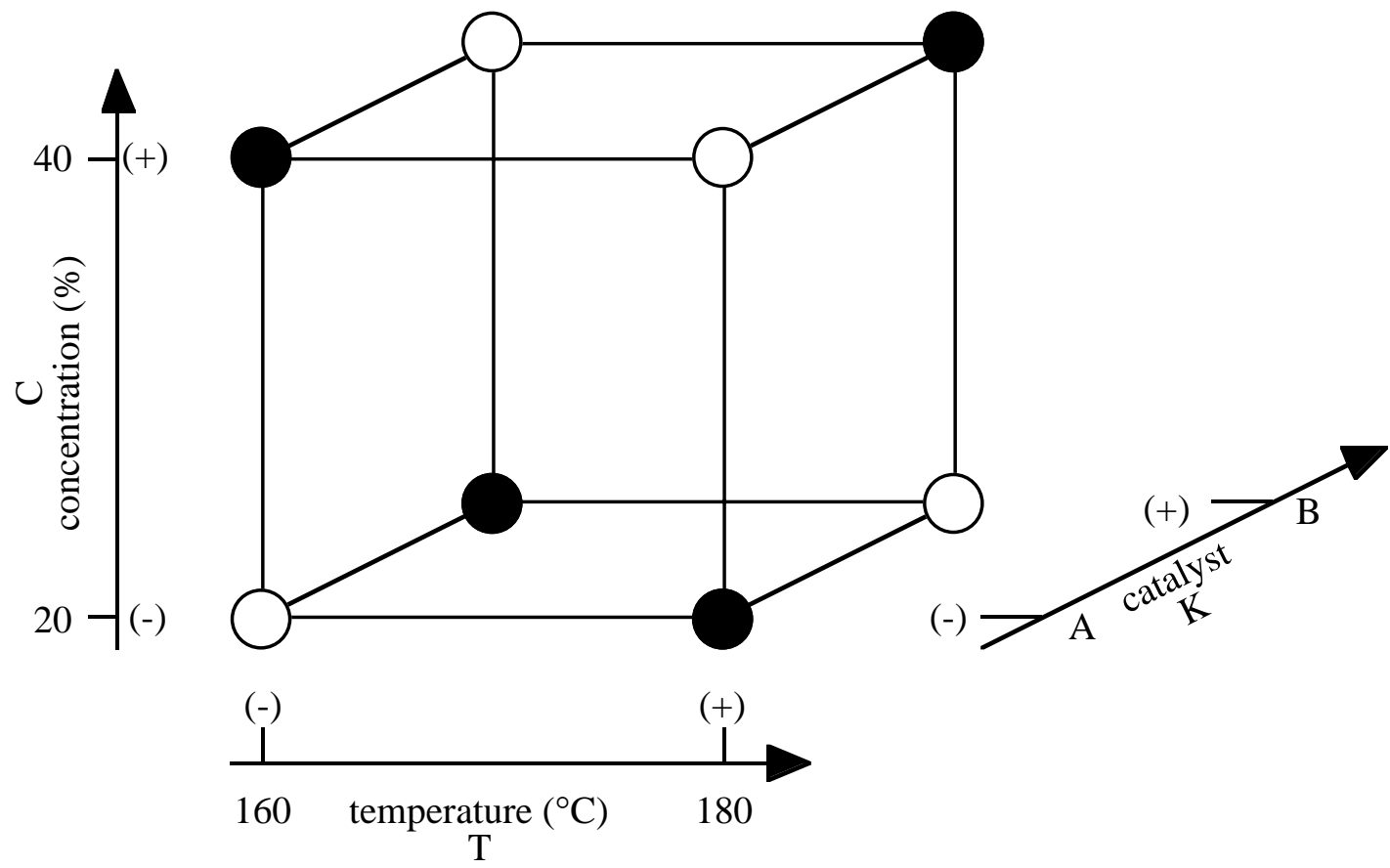

\section{Figure 1: Graphical Representation of a $2^{3}$ Factorial Design}

Figure 1 pictorially represents a factorial design with concentration, temperature, and catalyst as the factors, each with a high and a low setting. Table 1 lists the eight experiments for the full factorial design, where (+) represents the high setting and (-) represents the low setting. Note that these experiments should be run in a random order so time dependent effects do not become coupled with one of the factors. For example, if the set of experiments shown in Table 1 were run in the order shown, it would be difficult to discern effects associated with time from those associated with varying concentration. 


\section{Table 1: Full 23 Factorial Design}

\begin{tabular}{cccc}
\hline Experiment & $\mathrm{C}$ & $\mathrm{T}$ & $\mathrm{K}$ \\
\hline 1 & + & + & + \\
2 & + & + & - \\
3 & + & - & + \\
4 & + & - & - \\
5 & - & + & + \\
6 & - & + & - \\
7 & - & - & + \\
8 & - & - & - \\
\hline
\end{tabular}

\section{2 Fractional Factorial Designs}

Fractional factorial designs retain the properties of a factorial design but use fewer (by a factor of 2) observations. With the reduced number of experiments, the effects of the interactions become coupled. In order to have a well balanced factorial design, it is important understand which effects are coupled. For example, a well balanced half factorial design is represented in Figure 1 with the black and white points. In this design, the mean effect is coupled with the three way interaction between concentration, temperature, and catalyst $\left(\mathrm{C}^{*} \mathrm{~T}^{*} \mathrm{~K}\right)$, while the concentration is coupled with the interaction $\mathrm{T}^{*} \mathrm{~K}$, temperature is coupled with $\mathrm{C}^{*} \mathrm{~K}$, and catalyst is coupled with $\mathrm{C}^{*} \mathrm{~T}$. This means that, with this half factorial design, during data analysis it is impossible to distinguish between temperature and the concentration/catalyst interaction. A good design will confound the main effect (zero order interaction) with high order interactions.

As a rule, the number of $i^{\text {th }}$ order interactions in a $2^{\mathrm{k}}$ design is

$$
\left(\begin{array}{l}
k \\
i
\end{array}\right)=\frac{k !}{i !(k-i) !} .
$$

In the example above where $\mathrm{k}=3$,

$$
\begin{aligned}
& \text { \# zero order interactions }=\left(\begin{array}{l}
3 \\
0
\end{array}\right)=\frac{3 !}{0 !(3) !}=1 \\
& \# \text { first order interactions }=\left(\begin{array}{l}
3 \\
1
\end{array}\right)=\frac{3 !}{1 !(2) !}=3 \\
& \# \text { second order interactions }=\left(\begin{array}{l}
3 \\
2
\end{array}\right)=\frac{3 !}{2 !(1) !}=3 \\
& \# \text { third order interactions }=\left(\begin{array}{l}
3 \\
3
\end{array}\right)=\frac{3 !}{3 !(0) !}=1
\end{aligned}
$$

where the zero order interaction is the average response for all factors or main effect, equivalent to the constant term in the model. In order to study only the constant and first 
order interactions for the $2^{3}$ example, four experiments are required and the half factorial design is adequate. If we wish to study also the second order interactions, seven runs are required. A fractional factorial design only generates designs of powers of two, so an alternative method such as D-optimal design is required to optimize this experiment.

As a second example, let's consider a $2^{5}$ factorial design. Another useful tool is the Pascal triangle, shown in Figure 2.

Each row of the triangle represents the degrees of freedom associated with a successive factorial design, so the second row

\begin{tabular}{|ccccccccccccc|}
\hline & & & & & 1 & & & & & \\
& & & 1 & & 2 & & 1 & & & \\
& & 1 & & 3 & & 3 & & 1 & & \\
& 1 & & 4 & & 6 & & 4 & & 1 & \\
1 & & 5 & & 10 & & 10 & & 5 & & 1
\end{tabular}

Figure 2: Pascal's Triangle represents $2^{2}$ factorial design, while the fifth row is a $2^{5}$ factorial design. The first number in the row is the degrees of freedom for main effect, while the second number in the row is the degrees of freedom of first order interactions, etc. So the $2^{5}$ factorial design has one zero order interaction, five first order interactions, ten second order interactions, ten third order interactions, five fourth order interactions and one fifth order interaction totaling $2^{5}$, or 32 full factorial experiments. To study the main effect, first and second order interactions, sixteen runs are required, so a half factorial design is adequate. To select a well balanced design, it is important to understand which terms become coupled. A well balanced design will confound the main effect with the highest order interaction not considered significant. For this example, the design shown in Table 2 is well balanced with the confounded effects shown in Table 3.

Table 2: Full 23 Factorial Design

\begin{tabular}{cccccc}
\hline Experiment & 1 & 2 & 3 & 4 & 5 \\
\hline 1 & + & + & + & + & + \\
2 & + & + & + & - & - \\
3 & + & + & - & + & - \\
4 & + & + & - & - & + \\
5 & + & - & + & + & - \\
6 & + & - & + & - & + \\
7 & + & - & - & + & + \\
8 & + & - & - & - & - \\
9 & - & + & + & + & - \\
10 & - & + & + & - & + \\
11 & - & + & - & + & + \\
12 & - & + & - & - & - \\
13 & - & - & + & + & + \\
14 & - & - & + & - & - \\
15 & - & - & - & + & - \\
16 & - & - & - & - & + \\
\hline
\end{tabular}


Table 3: Compounded Interactions in a 23 Half Factorial Design

\begin{tabular}{ll}
\hline Effect & Compounded Interaction \\
\hline Main & $1 * 2 * 3 * 4 * 5$ \\
1 & $2 * 3 * 4 * 5$ \\
2 & $1 * 3 * 4 * 5$ \\
3 & $1 * 2 * 4 * 5$ \\
4 & $1 * 2 * 3 * 5$ \\
5 & $1 * 2 * 3 * 4$ \\
$1 * 2$ & $3 * 4 * 5$ \\
$1 * 3$ & $2 * 4 * 5$ \\
$1 * 4$ & $2 * 3 * 5$ \\
$1 * 5$ & $2 * 3 * 4$ \\
$2 * 3$ & $1 * 4 * 5$ \\
$2 * 4$ & $1 * 3 * 5$ \\
$2 * 5$ & $1 * 3 * 4$ \\
$3 * 4$ & $1 * 2 * 5$ \\
$3 * 5$ & $1 * 2 * 4$ \\
$4 * 5$ & $1 * 2 * 3$ \\
\hline
\end{tabular}

\section{3 D-Optimal Designs}

Design optimization techniques have been around for a long time. Many are graphically based and become difficult as the number of factors increases. With the use of computers, d-optimal design has become a very efficient design tool. The goal of d-optimal design is to select the subset of designs that minimizes the error of the model prediction. Consider a linear model where

$$
\mathbf{y}=\mathbf{X b}+\varepsilon
$$

where

$$
\begin{array}{ll}
\mathbf{y} & =n \times 1 \text { vector of measured values, } \\
\mathbf{X} & =n \times m \text { design matrix } \\
\mathbf{b} & =m \times 1 \text { vector of model coefficients, } \\
\varepsilon & =n \times 1 \text { vector of errors not explained by the model, } \\
n & =\text { number of measurements, and } \\
m & =\text { number of terms in the model. }
\end{array}
$$

The number of measurements $(n)$ must be greater than or equal to the number of terms in the model $(m)$. For example, for a linear model with three factors $(u, v, w)$ where the main effect and first order interactions are considered, 


$$
\begin{aligned}
& \mathbf{X}=\left[\begin{array}{ccccccc}
u_{1} & v_{1} & w_{1} & u_{1} v=v_{1} & u_{1} w_{1} & v_{1} w_{1} & 1 \\
u_{2} & \vdots & \vdots & \vdots & \vdots & \vdots & 1 \\
\vdots & \vdots & \vdots & \vdots & \vdots & \vdots & \vdots \\
\vdots & \vdots & \vdots & \vdots & \vdots & \vdots & \vdots \\
\vdots & \vdots & \vdots & \vdots & \vdots & \vdots & \vdots \\
\vdots & \vdots & \vdots & \vdots & \vdots & \vdots & \vdots \\
\vdots & \vdots & \vdots & \vdots & \vdots & \vdots & \vdots \\
u_{n} & v_{n} & w_{n} & u_{n} v_{n} & u_{n} w_{n} & v_{n} w_{n} & 1
\end{array}\right], \\
& \mathbf{y}=\left[\begin{array}{c}
y_{1} \\
y_{2} \\
\vdots \\
\vdots \\
\vdots \\
\vdots \\
\vdots \\
y_{n}
\end{array}\right], \\
& \mathbf{b}=\left[\begin{array}{c}
b_{1} \\
b_{2} \\
b_{3} \\
b_{4} \\
b_{5} \\
b_{6} \\
b_{7}
\end{array}\right], \\
&=\left[\begin{array}{c}
\varepsilon_{1} \\
\varepsilon_{2} \\
\vdots \\
\vdots \\
\vdots \\
\vdots \\
\varepsilon_{n}
\end{array}\right] .
\end{aligned}
$$

The least squares solution for the coefficients to Equation (2) is found by

$$
\begin{gathered}
\mathbf{X}^{T} \mathbf{y}=\mathbf{X}^{T} \mathbf{X b}, \\
\mathbf{b}=\left(\mathbf{X}^{T} \mathbf{X}\right)^{-1} \mathbf{X}^{T} \mathbf{y},
\end{gathered}
$$

The variance of the coefficients is

$$
\sigma_{b}=\left(\mathbf{X}^{T} \mathbf{X}\right)^{-1} \mathbf{X}^{T} \sigma_{y}
$$

and the variance of a predicted y value is

$$
\sigma_{y}=X^{T}\left(\mathbf{X}^{T} \mathbf{X}\right)^{-1} X^{T} \sigma_{y}
$$

where, for the design above,

$$
X=\left[\begin{array}{lllllll}
u & v & w & u v & u w & v w & 1
\end{array}\right] .
$$


To minimize the variance of the predicted values, we should select the measurement settings such that we minimize the $\left(\mathbf{X}^{T} \mathbf{X}\right)^{-1}$ matrix, where

$$
\left(\mathbf{X}^{T} \mathbf{X}\right)^{-1}=\frac{\operatorname{Adjoint}\left(\mathbf{X}^{T} \mathbf{X}\right)}{\operatorname{det}\left(\mathbf{X}^{T} \mathbf{X}\right)}
$$

One method of minimizing the $\left(\mathbf{X}^{T} \mathbf{X}\right)^{-1}$ matrix is then to maximize the determinant of the $\left(\mathbf{X}^{T} \mathbf{X}\right)$ matrix. This is known as d-optimal design.

There are other similar methods worth mentioning that also attempt to minimize the variance of the predicted values. They include minimizing the trace of the $\left(\mathbf{X}^{T} \mathbf{X}\right)^{-1}$ matrix and minimizing the maximum eigenvalues of the $\left(\mathbf{X}^{T} \mathbf{X}\right)^{-1}$ matrix.

\section{Data Analysis}

\subsection{Analysis of Variance and the F Statistic}

To determine which factors are significant, analysis of variance (ANOVA) is a useful tool. The ANOVA compares the level of influence of a factor to the overall scatter in the data. This ratio is called the F statistic. For substantially large F statistics, the factor can be considered significant. A percent significance can be obtained by referring to the $\mathrm{F}$ distribution which is often tabulated.

\subsection{Mallows $C_{p}$ Statistic}

Since the factors may be correlated, the ANOVA may produce erroneous results. In this case, Mallows $C_{p}$ statistic can be used. The $C_{p}$ statistic is an unbiased estimator of the total sum of squares of discrepancies (variance error plus bias error) of the candidate model from the true but unknown model, and is calculated by

$$
C_{p}=\frac{R S S_{p}}{s^{2}}-(n-2 p)
$$

where

$R S S_{p}=$ residual sum squared error from a candidate model containing $\mathrm{p}$ terms,

$p \quad=$ number of the terms in the candidate model,

$s^{2} \quad=$ residual mean squared error from the general model.

Since the expected value of $C_{p}$ is $p$, adequate models will show up as points close to $C_{p}=p$. The procedure is then to calculate the $C_{p}$ value for every combination of possible 
terms in the model. The model containing the significant terms will produce a $C_{p}$ value closest to $C_{p}=p$.

\subsection{Example}

I will use the $2^{3}$ full factorial design shown in Table 4 as an illustrative example. The three factors are humidity $(\mathrm{H})$, temperature $(\mathrm{T})$, and pressure $(\mathrm{P})$. A (-1) setting represents the low setting, while a (1) represents the high setting.

\section{Table 4: Sample Experiment}

\begin{tabular}{|c|c|c|c|c|}
\hline Experiment & Humidity & Temperature & Pressure & Response \\
\hline & 1 & 1 & - & 77 \\
\hline & 2 & 1 & & 67 \\
\hline & 3 & 1 & - & 28 \\
\hline & 4 & 1 & I & 32 \\
\hline & 5 & 1 & & 75 \\
\hline & 6 & 1 & & 73 \\
\hline & 7 & 1 & 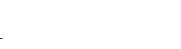 & 29 \\
\hline & 8 & 1 & 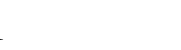 & 28 \\
\hline
\end{tabular}

The main effect is simply the average response, equal to 51.125. To determine the humidity effect, the main effect is subtracted from the average response when humidity is set high, so

$$
\mathrm{H} \text { effect }=\left(\frac{67+32+73+28}{4}\right)-51.125=-1.125 .
$$

The $\mathrm{H}^{*} \mathrm{~T}$ interaction is found by subtracting the main effect from the average response when the $\mathrm{H}^{*} \mathrm{~T}$ product is equal to 1 , so

$$
\mathrm{H}^{*} \mathrm{~T} \text { effect }=\left(\frac{77+32+75+28}{4}\right)-51.125=1.875 \text {. }
$$

Table 5 summarizes the results of each factor. These values could also be attained through a least squares fit of the data as shown in Equation 8. The fitted equation is

$$
\begin{aligned}
\text { resonse }= & 51.125-1.125 H-21.875 T+0.125 P+ \\
& 1.875 H^{*} T-0.875 T^{*} P+0.375 H^{*} P-1.625 H^{*} T^{*} P
\end{aligned}
$$

where each factor assumes a (1) or (-1) based on the setting shown in Table 4 . Note that the sum of each row is equal to the response so that there is no residual error. This is because the model contains eight terms and we are fitting the model with only eight points. Therefore, the fitted model will exactly represent the response. To perform an ANOVA on this experiment, we need an assessment of the overall scatter of the data. If we choose to include all interactions in the model, it is therefore not possible to perform an ANOVA. 
Table 5: Sample Experiment Results

\begin{tabular}{|c|c|c|c|c|c|c|c|c|c|c|}
\hline Experiment & Main & $\mathrm{H}$ & $\mathrm{T}$ & $\mathrm{P}$ & $\mathrm{H}^{* \mathrm{~T}}$ & $\mathrm{~T}^{*} \mathrm{P}$ & $\mathrm{H} * \mathrm{P}$ & $\mathrm{H} * \mathrm{~T} * \mathrm{P}$ & sum & Residuals \\
\hline 1 & 51.125 & 1.125 & 21.875 & -0.125 & 1.875 & -0.875 & 0.375 & 1.625 & 77 & 0 \\
\hline 2 & 51.125 & -1.125 & 21.875 & -0.125 & -1.875 & -0.875 & -0.375 & -1.625 & 67 & 0 \\
\hline 3 & 51.125 & 1.125 & -21.875 & -0.125 & -1.875 & 0.875 & 0.375 & -1.625 & 28 & 0 \\
\hline 4 & 51.125 & -1.125 & -21.875 & -0.125 & 1.875 & 0.875 & -0.375 & 1.625 & 32 & 0 \\
\hline 5 & 51.125 & 1.125 & 21.875 & 0.125 & 1.875 & 0.875 & -0.375 & -1.625 & 75 & 0 \\
\hline 6 & 51.125 & -1.125 & 21.875 & 0.125 & -1.875 & 0.875 & 0.375 & 1.625 & 73 & 0 \\
\hline 7 & 51.125 & 1.125 & -21.875 & 0.125 & -1.875 & -0.875 & -0.375 & 1.625 & 29 & 0 \\
\hline 8 & 51.125 & -1.125 & -21.875 & 0.125 & 1.875 & -0.875 & 0.375 & -1.625 & 28 & 0 \\
\hline $\begin{array}{l}\text { sum of } \\
\text { squares }\end{array}$ & 20910.13 & 10.125 & 3828.125 & 0.125 & 28.125 & 6.125 & 1.125 & 21.125 & 24805 & 0 \\
\hline
\end{tabular}

To perform an ANOVA, we must collapse the model on terms we believe to be insignificant. Based on the fitted coefficients, I chose to collapse the model on $\mathrm{P}, \mathrm{H}^{*} \mathrm{P}$, and $\mathrm{H}^{*} \mathrm{~T} * \mathrm{P}$. Now the fitted model becomes

$$
\text { resonse }=51.125-1.125 H-21.875 T+1.875 H^{*} T-0.875 T^{*} P .
$$

Since the model now has five terms, the least squares fit of all eight data points does not exactly fit the measured data. The residual errors are shown in Table 6.

Table 6: Sample Experiment Collapsed Design

\begin{tabular}{ccrrrrrr}
\hline Experiment & Main & \multicolumn{1}{c}{$\mathrm{H}$} & \multicolumn{1}{c}{$\mathrm{T}$} & \multicolumn{1}{c}{$\mathrm{H} * \mathrm{~T}$} & \multicolumn{1}{c}{$\mathrm{T} * \mathrm{P}$} & \multicolumn{1}{l}{ sum } & Residuals \\
\hline 1 & 51.125 & 1.125 & 21.875 & 1.875 & -0.875 & 75.125 & 1.875 \\
2 & 51.125 & -1.125 & 21.875 & -1.875 & -0.875 & 69.125 & -2.125 \\
3 & 51.125 & 1.125 & -21.875 & -1.875 & 0.875 & 29.375 & -1.375 \\
4 & 51.125 & -1.125 & -21.875 & 1.875 & 0.875 & 30.875 & 1.125 \\
5 & 51.125 & 1.125 & 21.875 & 1.875 & 0.875 & 76.875 & -1.875 \\
6 & 51.125 & -1.125 & 21.875 & -1.875 & 0.875 & 70.875 & 2.125 \\
7 & 51.125 & 1.125 & -21.875 & -1.875 & -0.875 & 27.625 & 1.375 \\
8 & 51.125 & -1.125 & -21.875 & 1.875 & -0.875 & 29.125 & -1.125 \\
\hline sum of & 20910.125 & 10.125 & 3828.125 & 28.125 & 6.125 & 24805.000 & 22.375 \\
squares & & & & & & & \\
\hline
\end{tabular}

The collapsed design produces the ANOVA shown in Table 7. The F statistic is found by dividing the mean squared error for each factor by the mean squared error for the residuals. The mean squared error is equal to the sum of squares (shown in Table 6) by the degrees of freedom. Each term in the model accounts for one degree of freedom, totaling five degrees of freedom. Since we have eight measurements, the residuals account for the remaining three degrees of freedom. The $\mathrm{p}$ value is obtained by referring to a table and looking up the corresponding $\mathrm{F}$ statistic with a degree of one in the numerator for the coefficient and a degree of freedom of three in the denominator for the residuals. The significance represents the likelihood that the term is significant. According to the ANOVA, the constant and the 
temperature are significant at $100 \%$ confidence level, while the $\mathrm{H}^{*} \mathrm{~T}$ interaction is 92.6 percent significant, etc.

Table 7: Sample Experiment ANOVA

\begin{tabular}{lrrrr}
\hline & Coefficient & Mean Squared Error & F Statistic & Significance \\
\hline Constant & 51.125 & 20910.130 & 2803.592 & 1.000 \\
Humidity & -1.125 & 10.125 & 1.358 & 0.836 \\
Temperature & -21.875 & 3828.125 & 513.268 & 1.000 \\
$\mathrm{H}^{* \mathrm{~T}}$ & 1.875 & 28.125 & 3.771 & 0.926 \\
$\mathrm{~T} * \mathrm{P}$ & -0.875 & 6.125 & 0.821 & 0.784 \\
\hline
\end{tabular}

Table 8: Sample Experiment $C_{p}$ Statistic

\begin{tabular}{lrl}
\hline \# Terms & \multicolumn{1}{c}{$\mathrm{C}_{\mathrm{p}}$} & Terms \\
\hline 1 & 516.218 & constant \\
2 & 4.950 & constant, T \\
3 & 3.179 & constant, T, H*T \\
4 & 3.821 & constant, H, T, H*T \\
5 & 5.000 & constant, H, T, H*T, T*P \\
\hline
\end{tabular}

Since some of the terms are correlated, these results may be erroneous. Therefore, I have also calculated the $C_{p}$ statistic for every possible combination of terms. The minimum $C_{p}$ values are reported in Table 8. In this example, the successive addition of terms reflects the results from the ANOVA. There are two methods used to select the best model. The first method is to select the model with the minimum $C_{p}$ value, while the second method selects the model with $C_{p}$ closest to the number of terms (with the exception of the model containing all terms, because $C_{p}$ will always equal the number of terms). For this example, the model containing three terms and the model containing four terms are equally close to the number of terms. Therefore, I would select the model with three terms since $C_{p}$ is a minimum. The significant terms are therefore the constant (or main effect), temperature, and the humidity/temperature interaction. This corresponds to the ANOVA result at $90 \%$ confidence level.

\section{Single Point Diamond Turning Experiments}

The goal of these experiments is to determine optimum cutting factors for the machining of silicon optics. We have completed the first step of identifying the factors, settings, and outputs. The three outputs are tool wear, subsurface damage and surface finish. The inputs are shown in Table 9. 
Table 9: Factors and Settings for Turning of Silicon Experiments

\begin{tabular}{|c|c|}
\hline Factor & Settings \\
\hline workpiece velocity & $\begin{array}{l}6 \mathrm{ft} / \mathrm{min} \\
500 \mathrm{ft} / \mathrm{min} \\
1000 \mathrm{ft} / \mathrm{min}\end{array}$ \\
\hline feedrate & $\begin{array}{l}20 \mu " / r e v \\
40 \mu " / r e v \\
80 \mu " / r e v \\
160 \mu " / r e v \\
250 \mu " / r e v \\
960 \mu " / r e v\end{array}$ \\
\hline depth of cut & $\begin{array}{l}40 \mu ” \\
80 \mu ” \\
160 \mu ” \\
200 \mu ”\end{array}$ \\
\hline nose radius & $\begin{array}{l}0.03 " \\
0.06 "\end{array}$ \\
\hline tool rake & $\begin{array}{l}0^{\circ} \\
20^{\circ} \\
40^{\circ}\end{array}$ \\
\hline skewed rake & $\begin{array}{l}0^{\circ} \\
40^{\circ}\end{array}$ \\
\hline cutting fluids & $\begin{array}{l}\text { glycol, polyalkaline } \\
\text { glycol, polyalkaline w/water } \\
\text { glycol, polyalkaline w/water and phosphate }\end{array}$ \\
\hline
\end{tabular}

A full factorial experiment would require the impractical number of 5184 total experiments. We therefore have decided to use experimental design. We have decided to first perform a screening design to determine which factors and interactions are significant. Once we determine the significance of each term, we will perform an RSM study where we will determine the optimum settings for the diamond turning process.

The screening experiments are the most efficient method to determine the significance of each term. For example, the velocity is not expected to influence the process. By including this factor in the screening experiments, we can be confident that its omission during the RSM study is legitimate. It may seem that, with a factor such as velocity where we have an intuition that it will not be significant, it may be more efficient to study this factor individually rather than including it in the matrix of screening experiments. This is an incorrect assumption. If we assume that velocity does not interact with any of the other settings, we would have to perform at least two separate experiments to determine if velocity has influence. With only two experiments, we have no repeated data to assure that the effects we have observed are significant rather than noise. Therefore, to be completely convinced, we would need to perform two more repeated tests. However, excluding the one factor from the screening matrix will only reduce the total number of experiments by 
one. This is accomplished by the fact that we vary more than one factor at a time in the screening experiments.

For the screening experiments, we have chosen to only study two settings (a high and low) for each factor with the exception of the cutting fluid, since the cutting fluid is a qualitative rather than quantitative factor. Based on experience, we have set the a priori model to include the main effect, all first order effects, and interactions between depth of cut and feedrate. We have also included chip thickness, which is a calculated value equal to

$$
C T=F R \sqrt{\frac{2 D O C}{N R}} .
$$

where the symbols are defined in Table 10. I have determined that fourteen experiments are required during screening, as shown in Table 10.

\section{Table 10: Degrees of Freedom for Turning of Silicon Experiments}

\begin{tabular}{lccc}
\hline \multicolumn{1}{c}{ Term } & Symbol & $\begin{array}{c}\text { Number of } \\
\text { Settings }\end{array}$ & Degrees of Freedom \\
\hline velocity & V & 2 & 1 \\
feedrate & FR & 2 & 1 \\
depth of cut & DOC & 2 & 1 \\
nose profile & NR & 2 & 1 \\
tool rake & TR & 2 & 1 \\
skewed rake & SR & 2 & 1 \\
cutting fluid & F & 3 & 1 \\
chip thickness & CT & ------------- & 1 \\
interaction & FR*DOC & ------------ & 1 \\
constant & & ----------- & 3 \\
residuals & & --- & 14 \\
\hline TOTAL & & & \\
\hline
\end{tabular}

Using d-optimal design, the optimum set of screening experiments are shown in Table 11. During the screening experiment, to reduce the testing time we have chosen to cut approximately one tenth the total length. The time required to perform the screening experiment is shown in the second to last column, while the time to cut the full length part is shown in the last column. Where possible, these experiments should be run in a random order so that time dependent effects are not compounded with the factors. However, it is not practically possible to randomize the order of the cutting fluid.

Once all the screening experiments have been complete, we will analyze the data and refine the model to include only the significant effects. We will then design the RSM experiments. We will use all the settings shown in Table 9 for the significant factors and design a reduced number of tests using d-optimal design. 
Table 11: Screening Experiments

\begin{tabular}{|c|c|c|c|c|c|c|c|c|c|c|}
\hline Exp. \# & $\begin{array}{c}\mathrm{V} \\
\mathrm{ft} / \mathrm{min}\end{array}$ & $\begin{array}{l}\text { FR } \\
\mu " / / r e v\end{array}$ & $\begin{array}{l}\text { DOC } \\
\mu \text { in }\end{array}$ & $\begin{array}{l}\mathrm{NR} \\
\text { in }\end{array}$ & $\begin{array}{l}\text { TR } \\
\text { deg }\end{array}$ & $\begin{array}{l}\text { SR } \\
\text { deg }\end{array}$ & $\mathrm{F}$ & $\begin{array}{c}\text { screen } \\
\text { time } \\
\text { hr }\end{array}$ & $\begin{array}{c}\text { full time } \\
\mathrm{hr}\end{array}$ & $\begin{array}{l}\text { CT } \\
\mu ”\end{array}$ \\
\hline 1 & 750 & 50 & 200 & 0.06 & 0 & 0 & gly/ poly & 0.65 & 4.53 & 4.08 \\
\hline 2 & 100 & 120 & 200 & 0.06 & 40 & 0 & gly/ poly & 2.02 & 14.15 & 9.80 \\
\hline 3 & 100 & 120 & 40 & 0.03 & 0 & 40 & gly/ poly & 2.02 & 14.15 & 6.20 \\
\hline 4 & 750 & 50 & 40 & 0.06 & 0 & 40 & gly/ poly & 0.65 & 4.53 & 1.83 \\
\hline 5 & 100 & 50 & 40 & 0.03 & 40 & 40 & gly/ poly & 4.85 & 33.96 & 2.58 \\
\hline 6 & 100 & 120 & 200 & 0.03 & 0 & 0 & gly/ poly/ h20 & 2.02 & 14.15 & 13.86 \\
\hline 7 & 750 & 120 & 40 & 0.03 & 40 & 0 & gly/ poly/ h20 & 0.27 & 1.89 & 6.2 \\
\hline 8 & 100 & 50 & 40 & 0.03 & 0 & 40 & gly/ poly/ h20 & 4.85 & 33.96 & 2.58 \\
\hline 9 & 750 & 120 & 200 & 0.06 & 0 & 40 & gly/ poly/ h20 & 0.27 & 1.89 & 9.80 \\
\hline 10 & 100 & 50 & 200 & 0.06 & 40 & 0 & gly/ poly/ h20 & 4.85 & 33.96 & 4.08 \\
\hline 11 & 750 & 50 & 200 & 0.03 & 0 & 0 & gly/ poly/ h20/ phos & 0.65 & 4.53 & 5.77 \\
\hline 12 & 100 & 50 & 40 & 0.06 & 40 & 0 & gly/ poly/ h20/ phos & 4.85 & 33.96 & 1.83 \\
\hline 13 & 100 & 120 & 40 & 0.06 & 0 & 40 & gly/ poly/ h20/ phos & 2.02 & 14.15 & 4.38 \\
\hline 14 & 750 & 120 & 200 & 0.03 & 40 & 40 & gly/ poly/ h20/ phos & 0.27 & 1.89 & 13.86 \\
\hline & & & & & & & & 30.24 & 211.71 & 29.92 \\
\hline
\end{tabular}




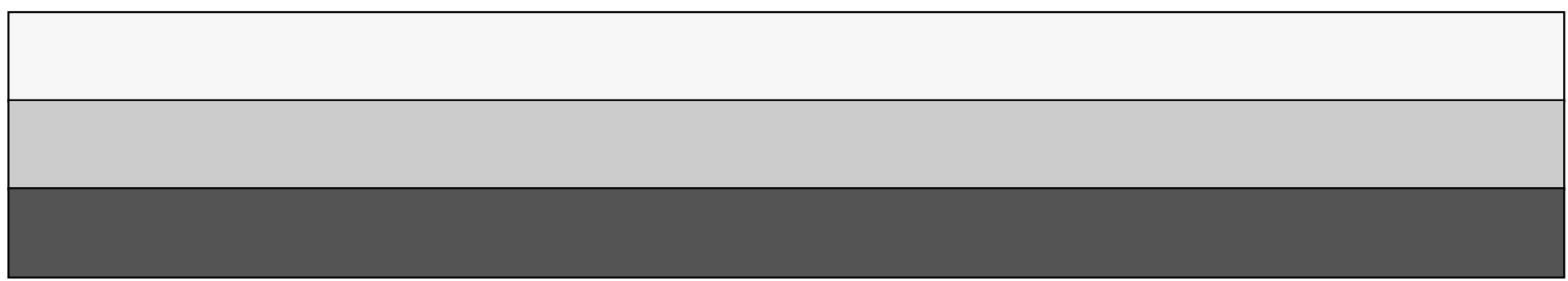

